\title{
Al Opponents with Personality Traits in Überpong
}

\author{
Carlos Delgado-Mata \\ Nibbo Studios \\ Michoacán 200-B, CP 20270 \\ Aguascalientes, México \\ Universidad Panamericana \\ Aguascalientes, México \\ Heriot Watt University \\ Edinburgh, Scotland \\ carlos@nibbo.net
}

\author{
Jesus Ibáñez-Martínez \\ Department of Technology \\ Universitat Pompeu Fabra \\ Barcelona \\ Catalunya \\ Spain \\ jesus.ibanez@upf.edu
}

\begin{abstract}
Nowadays, the video gaming experience is shifting from merely realistic to believable. The increasing graphic power of current graphic cards has made it possible to render near lifelike images. Unfortunately, the behaviour of the computer driven player and non-playing characters is often poor when compared to their visual appearance. In this sense, there has been a recent interest in improving the video gaming experience with novel Artificial Intelligence (AI) techniques. This paper presents a robotics inspired behavioural AI technique to simulate characters' personalities in an multi-award winning commercial video game.
\end{abstract}

\section{Categories and Subject Descriptors}

I.2 [Artificial Intelligence]: Applications and Expert Systems-Games

\section{General Terms}

Algorithms, Design

\section{Keywords}

Video game opponent, Artificial Intelligence, behavioural robotics, artificial personality trait

\section{INTRODUCTION}

The interest in Artificial Intelligence in Video Games has increased in recent years. The graphic power available in next generation console games (Nintendo's Wii, Sony's PS3 and Microsoft's XBox 360) and graphics cards allows the artist to create visually near life-like virtual worlds, virtual objects and virtual characters. However, the behaviour of the virtual beings that populate these environments, often lacks believability. This is made more notorious, when the behaviour is compared against pristine graphical models

Permission to make digital or hard copies of all or part of this work for personal or classroom use is granted without fee provided that copies are not made or distributed for profit or commercial advantage and that copies bear this notice and the full citation on the first page. To copy otherwise, or republish, to post on servers or to redistribute to lists, requires prior specific permission and/or a fee. The Second International Conference on Intelligent Technologies for Interactive Entertainment (ICST INTETAIN '08). January 8-10, 2008, Cancun, Mexico. Copyright 2008 ICST. ISBN 978-963-979913-4. (sadly, it is all-looks and no-brains). This mismatch aggravates the playability and believability of those virtual worlds. For that reason, there has been an interest in using traditional academic AI techniques (such as Neural Networks, Genetic Algorithms and Planning to name a few) within video games [4]. These techniques have been used instead (or in complement) of the good old Finite State Machines, that have been quintessential to video game's artificial intelligence. The novel approach presented herein is the use of behaviourally appealing opponents developed using an architecture inspired in behabioural robotics. Überpong, whose AI is the subject matter of this paper, is a video game that combines the genres of sports and action, and brings the classic game of Pong to the XXI century. To achieve this, several interesting algorithms of computational physics, collision detection and AI were developed.

The road map for this paper is as follows. Firstly, the state of AI in the videogaming industry is presented. Secondly, the use of behavioural inspired AI to provide different personality traits to different opponents is described. Thirdly, the implementation is presented. Fourthly, the results are discussed. Finally, the conclusion and future work is discussed.

\section{RELATED WORK}

Video games in particular, and virtual environments in general, have relied on artificial intelligence to create the 'illusion of life' in computer driven opponents and Non Playing Characters (NPC) for a few decades. Video games from previous generations had big resource restrictions. Therefore, the attention in providing computer driven opponents with behaviours that only use a very small fraction of the scarse available resources. Furthermore, those developers used just a fraction of those available resources for AI. Nevertheless, some developers accomplished behaviours which were remarkable; one such example is Pac-man, developed primarily by Toru Iwatani of Namco, in which each of the computer driven opponents (ghosts) has a particular behaviour to simulate a personality trait. One ghost chases Pac-man (which can be construed as brave). A second ghost wanders randomly (which can be construed as silly). The last two ghosts seem to work as a team, and their behaviour can be construed as team work. This appalling restriction does not longer exist and the amount of resources available to developers now is vast and thence more resources can be used for developing interesting AI for the computer driven 
opponents and NPCs. Some examples are mentioned next.

\subsection{Creatures}

This game was designed by Steve Grand. In this very interesting game (Artificial Life Simulation), each of the creatures (Norns), is instantiated out of genetical information (digital genes). This information can be transmitted to future generations by mating, and that does provide their means to evolve into creatures with unique characteristics (systems). Examples of the latter is the digestive system and the action selection mechanism, which is made up of a simple neural network. Creatures presented an interesting insight in artificial life creation [13] in video games.

\subsection{Black and White}

Peter Molyneux, of Lionhead Studios, produced the Black and White video game, in which Richard Evans developed complex AI for it. Techniques used therein furthered the AI seen in video games. The first novel AI technique used in Black and White is the use of Reinforcement Learning to teach the beasts (creatures) in the game. Teaching is performed by patting the back of the creature, if the player is happy with the creature's behaviour; or a by hitting it with a leach if the player is not pleased with the creature's behaviour. Also of interest is the influence of the player's decisions in the environment. If these are perceived to be good, the landscape is colourful; whereas, if these are perceived to be bad, the landscape turn into dark and evil. Other novel uses of AI that were introduced in this ground breaking game is the mouse gesture recognition used to cast spells, and finally, it is worth mentioning the use of psychologically plausible agents [6] with the implementation of a Belief-Desire-Intention agent architecture [21] for Non Player Characters.

\subsection{F.E.A.R (First Encounter Assault Recon)}

Jeff Orkin, of Monolith Productions, developed the game's AI for the F.E.A.R video game [17]. This game has been critically acclaimed and commercially successful. The AI presented in this game is one of the most interesting seen in video games. The novel AI used for this video game is a planner which complements other techniques that are normally used in video games, like Finite State Machines and the $\mathrm{A}^{*}$ algorithm for path planning. The novel use of this planner is that it simplifies the creation of interesting behaviours for NPCs or enemies.

Therein, a set of objectives are defined for the NPC, that are not coupled with the possible actions that the NPC can perform to achieve their goals. This makes the AI system configurable and manageable if the developer is to expand or change the NPC's repertoire of behaviours.

\subsection{Unreal Tournament}

Unreal Tournament (UT) provides a mean to configure a NPC behaviour via Unreal Script. Furthermore, an API (Gamebots), which [1] is a modification to UT, allows characters in the game to be controlled via network sockets connected to other programs (for a detailed description of Gamebots see [15]). These features have been so successful that the UT has become a tool to do academic AI research. As pointed out in [16], although the development of realistic virtual environments is an expensive and time-consuming enterprise that requires expertise in many areas, computer games provide us with a source of cheap, reliable, and flexible technology for developing our own virtual environment for research. In this sense, the current trend is to use the UT engine as a development platform. UT is being used, for instance, to develop storytelling systems [18] [22] [8] [14], and ai-based bots in general [16].

\subsection{Sims}

Another interesting example of what could be considered A-Life exploration in video games is that of the Sim's. According to Forbus and Wright (The Sims author) [12], the Sims' world is created on top of Edith. A simulation in Edith consists of a set of objects. The simulation is evolved by running each of these objects in turn. Every object has a set of local data and a set of behaviours. The local data provide the parameters of an object, including handler to objects it is related to. The set of behaviours consist of a procedure that implements it, a procedure that checks to see whether or not it is possible to accomplish it, and a set of advertisements that describe its properties in terms of what needs it will satisfy. Sims (not under direct player control) choose what to do by selecting, from all of the possible behaviours in all of the objects, the behaviour that maximises their current so-called state of happiness. Once they choose a behaviour, the procedure for that behaviour is then run in the thread of the Sim itself, so that it has access to that Sim's parameters in addition to those of its defining environment. Sometimes there are a number of intermediate objects to implement behaviours. For instance, Social Interaction is an object that is created and used when two Sims interact.

The games mentioned above borrowed techniques from AI and Artificial Life techniques normally used in academy. Thereby, these games have improved the perceived believability of the Non Playing Characters' behaviours. Similarly, the work proposed here, benefits from AI techniques used in other fields.

\section{AI TO PRODUCE PERSONALITY TRAITS}

To put this work in perspective, it should be noted that the model described in this section is applied to control the user's opponent in the video-game Überpong, which is somehow an evolution of the classic Pong. Pong, while not the first video game, was the first coin-op arcade game and the first mainstream video game that was available to almost everyone. Pong was invented by Ralph H. Baer in late 1960s, [2] and it was later licensed to Magnavox, which successfully marketed it. An arcade version of the game was developed by Atari, the company founded by Nolan Bushnel, in the 1970's. Pong was a pretty simple game with simple rules: hit the ball across the playing field and try your best to hit it past your opponents paddle on the other side (see figure 1). The origins of Pong lie with an abstract tennis game created with an old oscilloscope and some vacuum tubes by Willy Higinbotham, way back in 1958 [11].

\subsection{Background}

As stated before, Überpong is a video game that combines the sports and action genres. The game provides different characters with distinctive perceived personality profiles. The aim of the game, as in most sport games, is to score more points (goals) than the opponent. The game is played via a paddle (composed of a spring and two small spheres). The paddle spring is affected by spring physics, 


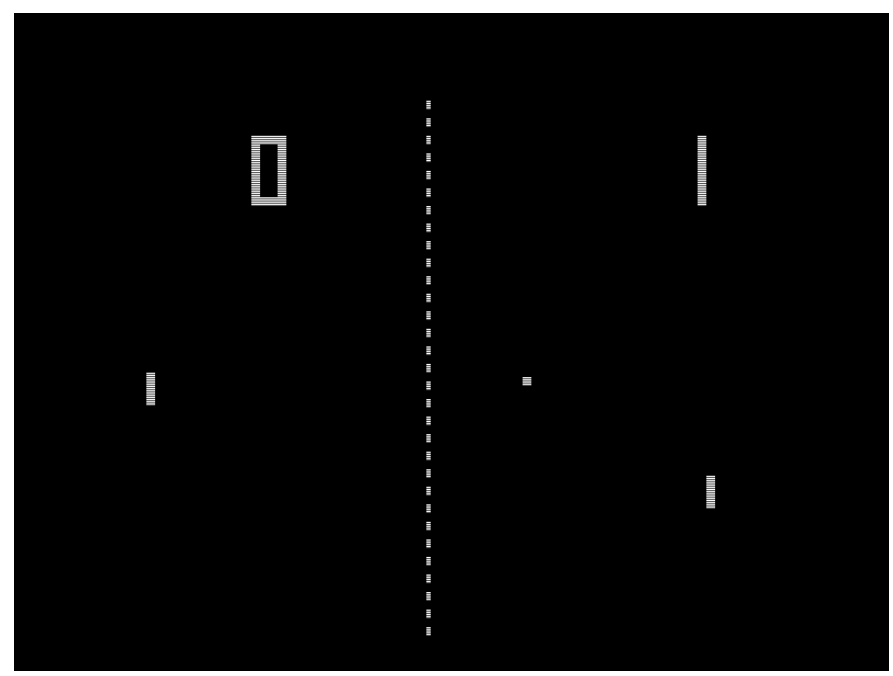

Figure 1: Original Pong

and thus, the ball can be affected in several ways. One is by tightening and loosening the tension of the spring as the ball collides with it. Another is by moving the paddle direction as the ball collides with paddle, and thus, change the direction of the ball by applying an effect. The computer driven player uses different strategies to affect the ball's acceleration and direction, depending on their personality trait. The players are also provided with special items (power ups) that can be used to affect the properties of the ball, one's own paddle, and the opponent's paddle. The correct use of these so-called special items can be vital to win a game. Therefore, it is important for the computer driven player to select and use an effective and compelling strategies to attack, defend, apply effects to the ball and use items and thus became a worthy and believable opponent.

\subsection{Behavioural Reactive Agents}

Research in behavioural based robotics has demonstrated that with the use of simple rules, the robot can perform complex and compelling behaviours. This field of research was inspired by the influential work of Braitenberg [5]. Some of his proposed robots display behaviours that can be construed as more complex than the simple rules used to implement them. For example, he named some of the robots' behaviour: 'love', 'fear' and 'aggression'. In a similar vein, Brooks proposed a horizontal architecture [7] for robots, this in turn is the inspiration for an architecture (Behavioural Synthesis Architecture) first used for cooperating robots [3]. This architecture, has been expanded to communicate emotions through artificial scents in virtual conspecific mammals, first described here [9]. Later this architecture was expanded to affect a flocking behaviour via an emotion architecture, which is described here [10].

Herein, a horizontal behavioural architecture not dissimilar to the aforementioned was developed and is used to drive the response of the computer player (AI of the game) and is described next.

\subsection{Behavioural Reactive Agents for Überpong}

The AI of Überpong is defined using four parameters. These are shown in figure 2 , where the stimuli is on the
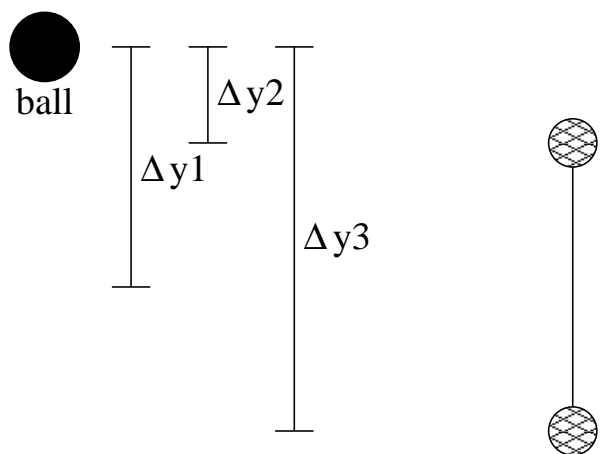

opponent

paddle

Figure 3: Following ball algorithm in Überpong

left; the four components are represented by the four black boxes; and the responses are represented by the arrows on the right.

1. How the computer driven opponent approaches the ball's destination.

2. First set of parameters to define the computer driven opponent personality profile.

3. Second set of parameters to define the computer driven opponent personality profile.

4. Third set of parameters to define the computer driven opponent personality profile.

While it is not the aim of this work to develop a "deep" cognitive personality model, or to implement a Social- Psychological Model, like the one found in [19]. For an excellent overview on Synthetic Personalities the reader is refered to [20]. The aim is instead, to develop a simple architecture to give perceived personality traits for video game opponents. This personality traits are defined by the manipulation of "simple" parameters. Simplicity in the manipulation of parameters is important for the level designer of the Überpong game.

\subsubsection{How the opponent approaches the ball's desti- nation.}

These parameters are used to define the strategy on how the AI driven player paddle's will approach the ball. There are three methods used. The first is simply follow the ball. This method computes the difference $(\Delta y)$ of the opponent's paddle position with the ball's position. The paddle position used depends on the status of the paddle's string, i.e. if the opponent's paddle spring is broken or not. This is shown in figure 3. If the spring is not broken the $\Delta y$ used will be $\Delta y 1$, if the spring is broken then $\Delta y$ would be the smaller of $\Delta y 2$ and $\Delta y 3$. Depending on the sign of $\Delta y$ and on the opponent's profile, defined in the XML described below, a velocity vector for the opponent's paddle is created. This vector is represented in to top reight arrow in figure 2

The second one is an erratic version of the first one. That is, noise is added to the velocity vector response. The third one is a predictive algorithm that computes where the ball is estimated to arrive when it crosses the plane described by 


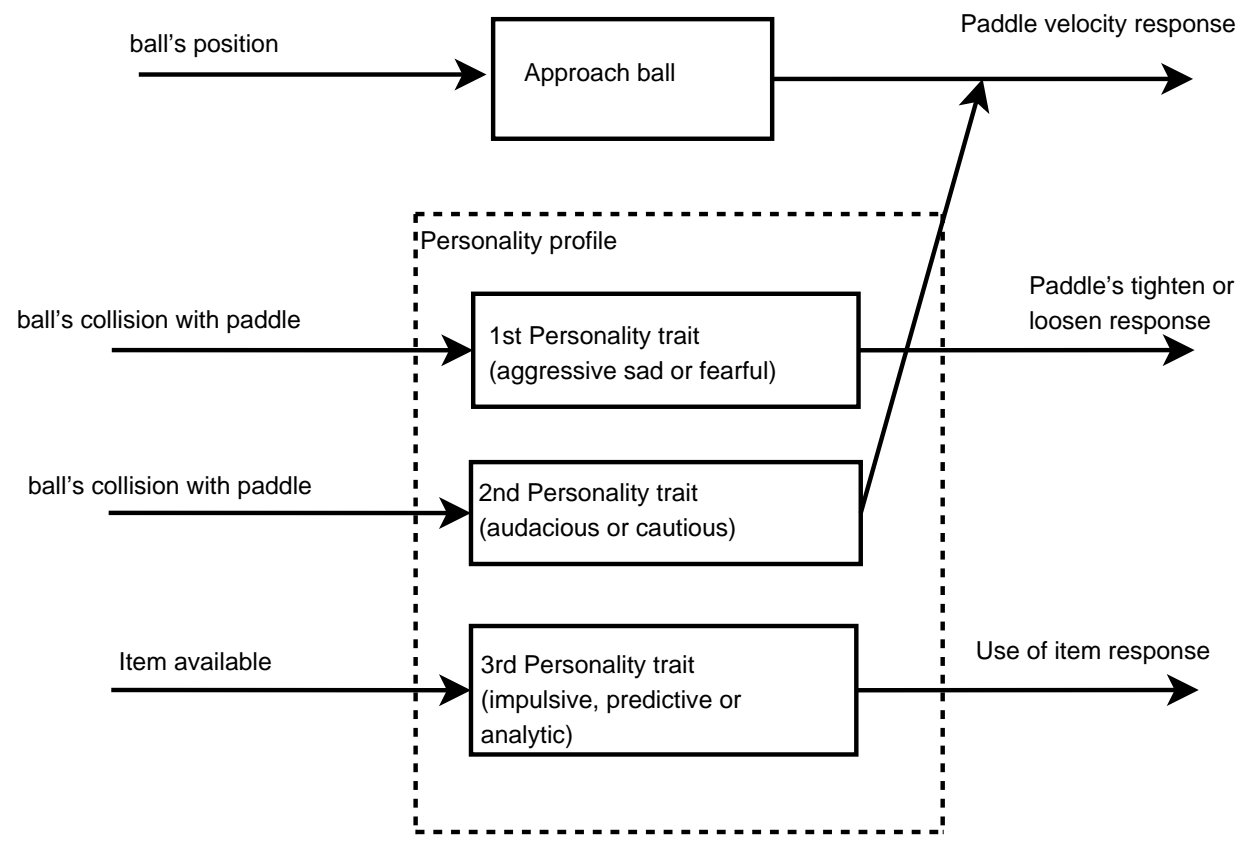

Figure 2: AI architecture of Überpong

the $\mathrm{x}$ position of the AI driven player, this algorithm uses our version of the death-reckoning algorithm shown in figure 4. $V$ is the velocity vector of the ball and $P$ is the opponent's paddle current position and $P^{\prime}$ is where the ball is going to cross the plane mentioned above. $\Delta y$ is the distance of the paddle position with the ball's calculated future position. With those values time is computed. With time the velocity of the paddle is affected, so that the opponent can reach the ball at the same time as the ball at $P^{\prime}$. The third strategy is used by the 'smarter' opponents.

In all the above-mentioned cases, there is a simple vision system, based on traditional Computer Graphics algorithms, to restrict vision between a near and a far plane. Parameters we also added to simulate decay in the quality of perception close the far plane.

An example of the parameters for this dimension is shown next.

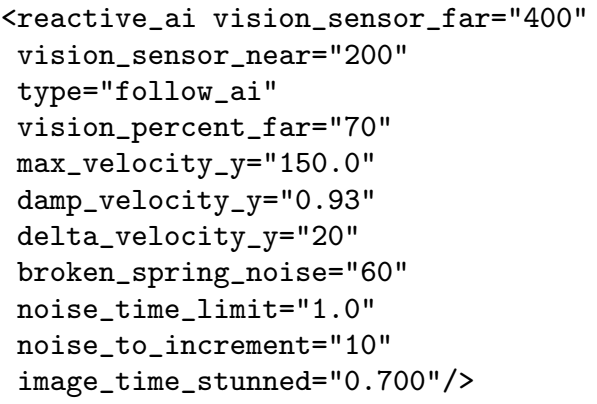

In this case, the above-listed parameters are used for a computer driven player with a follow ball strategy and with its vision constricted to the range of 200 to 400 pixels. The vision of the computer driven player decays close to the far limit, and in this particular case the decay is 70 per cent. The maximum velocity is restricted to 150.0 units per second. There are also parameters which are used when the

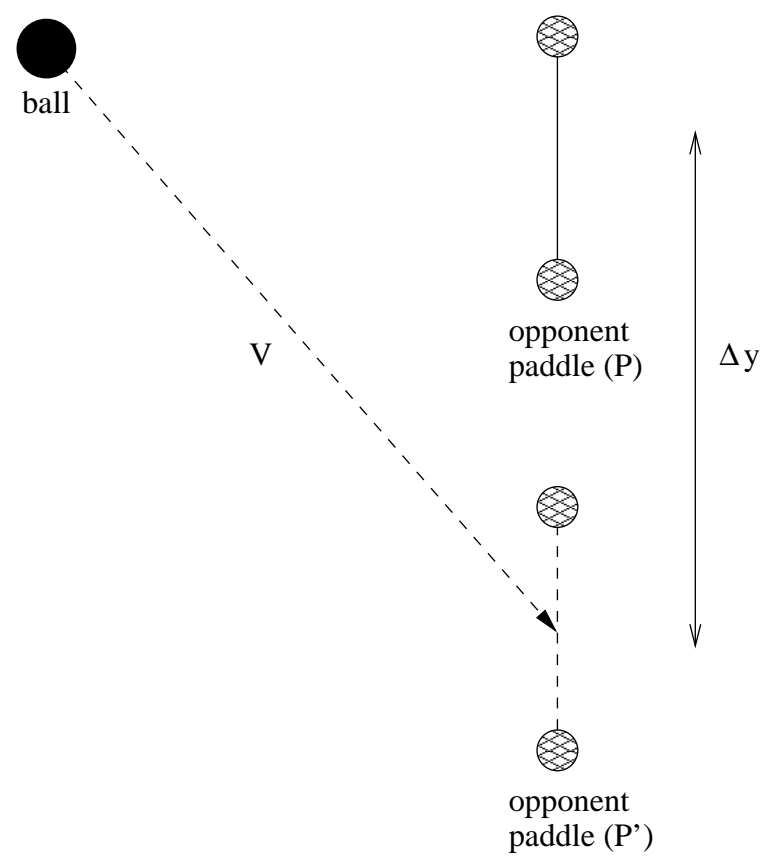

Figure 4: Predict ball's position algorithm in Überpong 
spring is broken. In the case presented here, the noise value, which affect the perception of the ball's $y$ position, is 60 units. There is a time limit value of 1.0 seconds, before the noise's value increments in 10 units every 1.0 seconds. This simulates fatigue in the computer driven opponent. The last parameter is used to stun the computer driven player in case that the opponent applies a swap image power up, and thus the computer driven opponent would react as a human driven player would. That is, the opponent is 'confused' for a few miliseconds.

\subsubsection{First Set of Parameters to define Personality}

These parameters define the personality traits: aggressive, sad and fearful. The strategy pursued to stretch and loosen of the paddle depends on which personality trait is chosen for the AI driven player. This is shown in figure 5 . The opponent with an aggressive personality trait, tries to accelerate the ball by loosening the paddle before the ball approaches it and then the opponent tightens the paddle as the ball is colliding with the paddle's spring. The fearful opponent tries to de-accelerate the ball by tightening the paddle before the ball approaches and then loosening the paddle as the ball collides, and the sad player just plays a safe game and keeps the paddle stretched all the time. An example of the parameters used is shown next. Depending on the strategy pursued by the computer driven opponent the ball's acceleration is affected using spring physics.

$$
a=-k \frac{\Delta x}{m}
$$

Where $k$ is the spring constant, which depends on the opponent's profile, $\Delta x$ depends if the spring was tightened or loosened and $m$ is the ball's mass. The mass is useful as there is an special effect that increases the ball's size and thence it's mass.

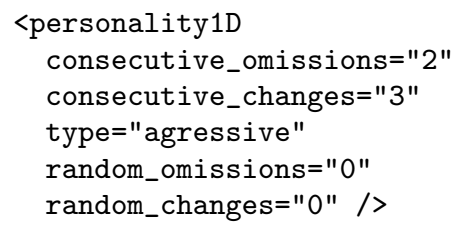

The parameters listed above are for a computer driven player with an aggressive personality trait that will try to accelerate the ball three consecutive times followed by two consecutive times where it does not try to accelerate the ball.

\subsubsection{Second Set of Parameters to define Personality}

These parameters define the personality traits audacious and cautious. Depending on the personality trait selected the opponent moves the paddle up or down as they receive the the ball to apply a ball effect to their response and therefore change the flow of the expected answer, as shown in figure $6 . V$ is a vector which will affect the final velocity of the paddle, in figure 2 this vector is represented by the third topmost arrow on the right (responses). The opponent with an audacious personality trait applies ball effects, whilst the AI driven opponent with a cautious personality trait does not. An example of the parameters is shown next.
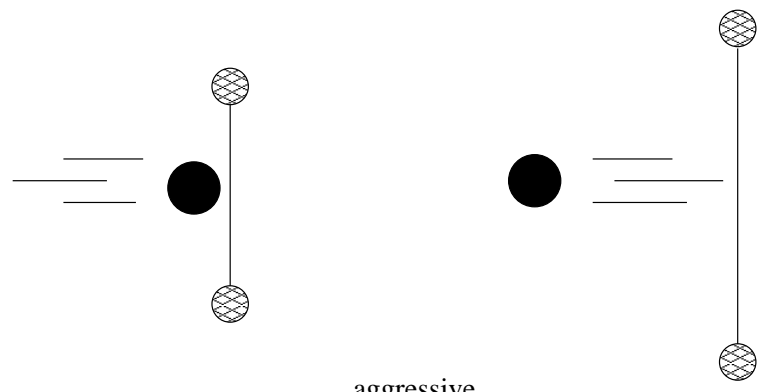

aggressive
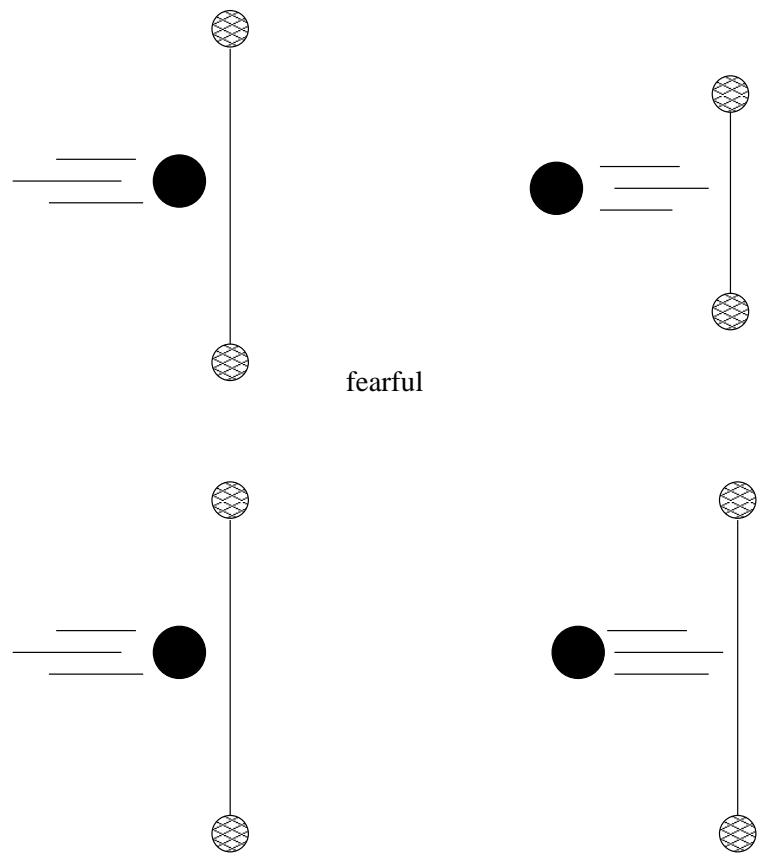

sad

Figure 5: First personality trait for the AI opponent

$<$ personality2D

delta_change="0.0" 

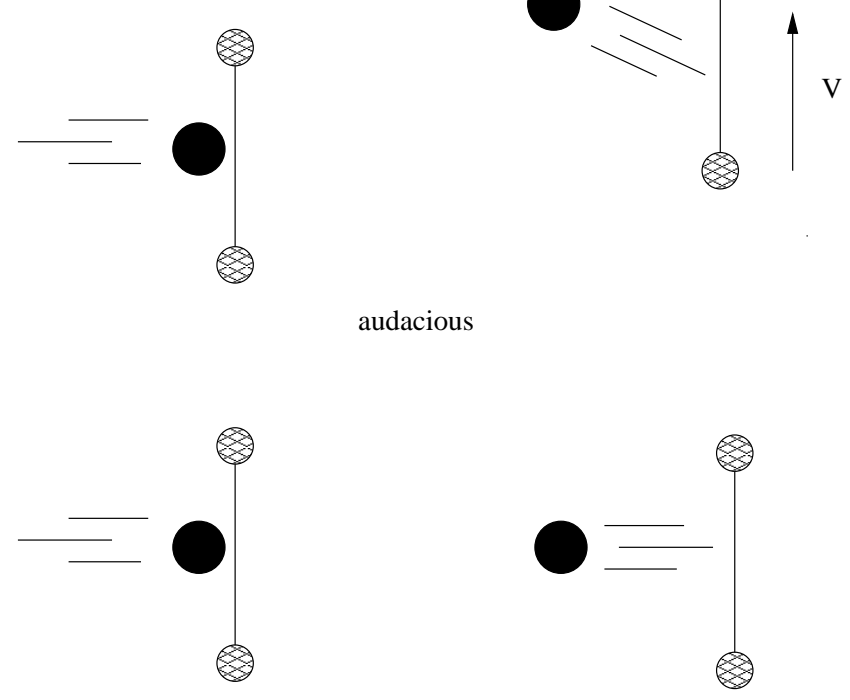

cautious

Figure 6: Second personality trait the AI opponent

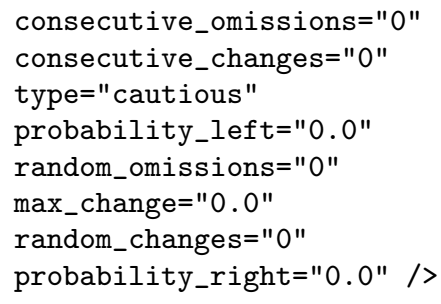

The above-listed parameters are used for a computer driven player with a cautious personality trait and thus it will not attempt to change the ball's direction by applying an effect to the ball.

\subsubsection{Third Set of Parameters to define Personality}

These parameters define the personality traits impulsive, predictable and analytic. These parameters define the strategy of how the opponent will use the power-ups available to them. The opponent with an impulsive personality trait uses the power-up as they receive it (a knee jerk reaction), the opponent with a predictable personality trait will wait $x$ seconds to apply the power up. The opponent with an analytic personality trait 'analyses' the moment to cause the most damage to the opponent. An example of the parameters is shown next.

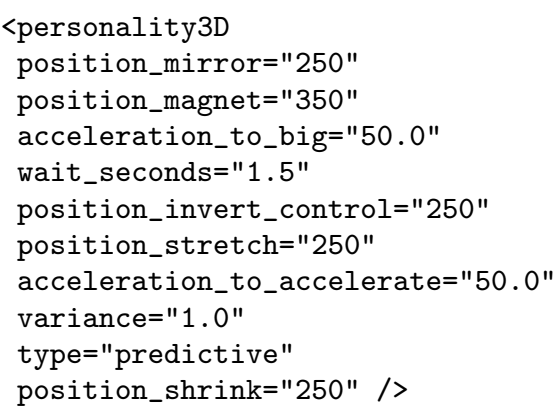

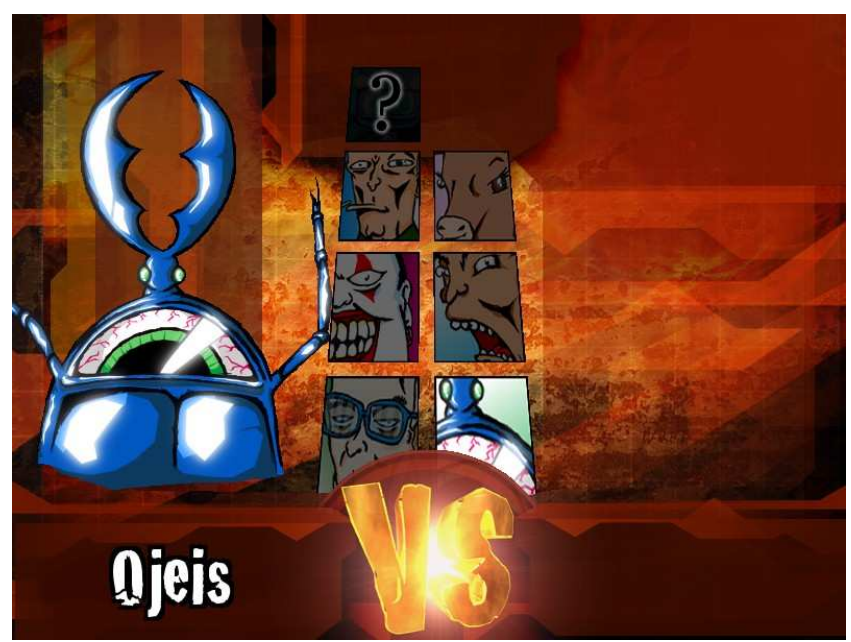

Figure 7: Some Characters in Überpong

The above-listed parameters are used for a computer driven player with a predictive personality trait and thus it will attempt to use an item (power-up) after 1.5 seconds of receiving the opponent, the other parameters are shown for the case of an analytic personality trait. These parameters are used as a trigger to apply the item to the opponent. For example, the parameter position_invert_control = "250", dictates that the invert control item will be released when the ball is 250 units or closer to the player's paddle $x$ position.

\section{IMPLEMENTATION}

The video game Überpong, whose Artificial Intelligence to drive the computer player is described in this paper, was developed by Nibbo Studios. This game has won several awards; one is the first place (obtained with an alfa version) for a video game developed by company in the 2006 video game development competition organised by the Mexican chapter of the International Game Developers Association, the Electronic Gamming Show, and the Economy Secretariat. It has also been praised by two Independent Game Festival judges, and has been selected as a semifinalist in the international 2nd Indie Game Developers Showcase in August 2007.

In Überpong, there are different characters that the player and the opponent can select, some of them are shown in figure 7. Each character has different properties that affect their behaviour and performance. These depend on their personality traits. Relevant to the argument of this paper are the properties to create a personality profile with the AI personality traits described before. In figure 8 the opponent prepares its move. In figure 9 the opponent has returned the ball.

\section{RESULTS}

The final version of the AI, which is described in this document, is the product of issues raised during the extensive beta testing period and the valuable comments from several judges of the game competitions that Überpong has taken part. The players let us know, via the beta testing formulaire, how they perceived the opponents behaviour, and how that affected their experience, during the playing time of 


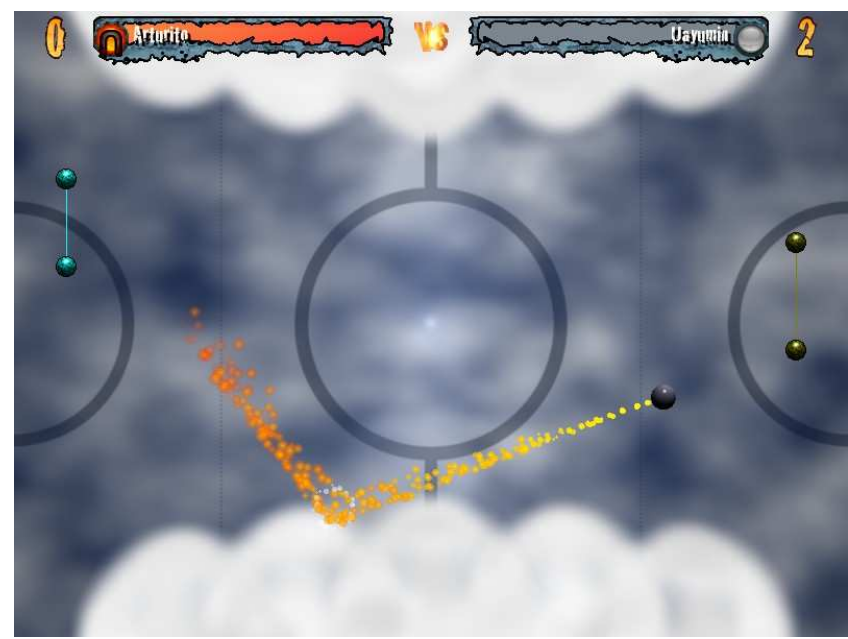

Figure 8: Opponent (on the right) is preparing its move the video game. In previous versions, they stated that the AI was "lacking something", that the computer driven opponent's behaviour "was not realistic", that the behaviour "looked odd", that the "opponent's strategy was not believable", or worse that "the opponent was dumb". On the other hand, some reactions from the final version are "the opponent caught me off guard -It was just like playing against a human player", "the opponent really knew when to use the special item". There is a special mode of play when two computer driven players compete against each other and when it was shown to some people in an event the reaction was of disbelief - "Surely this is a video, there is no way computer driven players can play like that". It can be seen from these comments that the AI architecture described above has greatly enhanced the user's experience by making the opponent more believable and hence more engaging and fun to play against.

\section{CONCLUSION AND FUTURE WORK}

The recent shift from graphical realism to believability in video games is the reason of the recent interest in the use of academic artificial intelligence to manage computer driven opponents and non-playing characters. In this paper, an approach (inspired in behavioural robotics and behavioural reactive agents) for computer driven players with personalities is presented. This architecture was developed and is used successfully in a commercial video game. This video game has caught the attention of players and reviewers. Tests will be carried out to find if the users detect the different personality traits of a computer driven character. These tests would also help to find out if the players perceive that the computer driven player's behaviour is plausible or if they just perceive that its behaviour is plausible, but they do not detect the different personality traits.

\section{ACKNOWLEDGEMENTS}

The authors would like to thank the rest of the developers and artists involved in Überpong. Special thanks to Oscar Guillén (creative lead) and Pier Guillén (Programming lead).

\section{REFERENCES}

[1] R. Adobbati, A. N. Marshall, A. Scholer, S. Tejada, G. A. Kaminka, S. Schaffer, and C. Sollitto. Gamebots: A 3d virtual world test-bed for multi-agent research. In Second International Workshop on Infrastructure for Agents, $M A S$, and Scalable $M A S$, Montreal, Canada, 2001.

[2] R. H. Baer. Television gaming apparatus and method. U.S. Pattent 3,659,285, April 1972.

[3] D. P. Barnes. A behaviour synthesis architecture for cooperant mobile robot. In J. O. Gray and D. G. Caldwell, editors, Advanced Robotics and Intelligent Machines, pages 295 - 314. IEE Control Engineering Series 51, United Kingdom, 1996.

[4] D. M. Bourg and G. Seemann. AI for Game Developers. O'Reilly Media, Inc., 2004.

[5] V. Braitenberg. Vehicles: Experiments in Synthetic Psychology. Papermac, Oxford, United Kingdom, 1984.

[6] M. E. Bratman. Intention, Plans, and Practical Reason. CSLI Publishing, USA, 1987. 
[7] R. A. Brooks. A robust layered control system for a mobile robot. IEEE Journal of Robotics and Automation, 2(1):14-23, 1986.

[8] M. Cavazza, J.-L. Lugrin, S. Hartley, P. Libardi, M. J. Barnes, M. L. Bras, M. L. Renard, L. Bec, and A. Nandi. Intelligent virtual environments for virtual reality art. Computers \& Graphics, 29(6):852 - 861, 2005.

[9] C. Delgado-Mata and R. Aylett. Communicating emotion in virtual environments through artificial scents. In E. de Antonio and R. Aylett, editors, LNCS Intelligent Virtual Agents, Madrid, Spain, 2001. Springer-Verlag.

[10] C. Delgado-Mata, J. Ibanez, and R. Aylett. Let's run for it: Conspecific emotional flocking triggered via virtual pheromones. In A. Butz, A. Krüger, and P. Oliver, editors, LNCS Smart Graphics 2003, pages 131-140, Heidelberg, Germany, 2003. Springer-Verlag.

[11] R. DeMaria and J. L. Wilson. High Score! The Illustrated History of Electronic Games. Osborne/McGraw-Hill, 2002.

[12] K. D. Forbus and W. Wrigth. Some notes on programming objects in the sims. Technical report, Northwestern University, 2001.

[13] S. Grand. Creatures:an exercise in creation. IEEE Intelligent Systems Magazine, July 1997.

[14] J. Ibánez, R. Aylett, and R. Ruiz-Rodarte. Storytelling in virtual environments from a virtual guide perspective. Journal of Virtual Reality, Springer, 7(1):30-42, December 2003. Especial Edition on Storytelling in Virtual Environments.

[15] G. A. Kaminka, M. M. Veloso, S. Schaffer, C. Sollitto, R. Adobbati, A. N. Marshall, A. Scholer, and S. Tejada. Gamebots: a flexible test bed for multiagent team research. Communications of the ACM, 45(1), January 2002.

[16] J. E. Laird. Research in human-level ai using computer games. Communications of the ACM, 45(1 SPECIAL ISSUE: Game engines in scientific research), January 2002.

[17] J. Orkin. Three states and a plan: The a.i. of f.e.a.r. In Game Developers Conference, San Jose, California, 2006. CMP Game Group.

[18] J. Robertson and J. Oberlander. Ghostwriter: Educational drama and presence in a virtual environment. Journal of Computer Mediated Communication, 8(1), 2002.

[19] D. Rousseau and B. Hayes-Roth. A social-psychological model for synthetic actors. In Creating Personalities for Synthetic Actors, pages 165-172, 1998.

[20] R. Trappl and P. Petta, editors. Creating Personalities for Synthetic Actors. Springer Verlag, 1997. LNAI 1195.

[21] M. Wooldridge. Reasoning About Rational Agents. MIT Press, USA, 2000.

[22] R. M. Young. An overview of the mimesis architecture: Integrating intelligent narrative control into an existing gaming environment. In The Working Notes of the AAAI Spring Symposium on Artificial Intelligence and Interactive Entertainment, Stanford, CA, March 2001. 